\title{
The relationship between motor milestone achievement and childhood motor deficits in children with Attention Deficit Hyperactivity Disorder (ADHD) and children with Developmental Coordination Disorder
}

\author{
Jenna Lee $^{\mathrm{a}, 1}$, Leighanne A. Mayall ${ }^{\mathrm{a}, 1}$, Kathryn E. Bates ${ }^{\mathrm{b}}$, Elisabeth L. Hill ${ }^{\mathrm{c}}$, \\ Hayley C. Leonard ${ }^{\mathrm{a}}$, Emily K. Farran ${ }^{\mathrm{a}, *}$ \\ ${ }^{a}$ University of Surrey, Guildford, Surrey, GU2 7XH, UK \\ ${ }^{\mathrm{b}}$ UCL Institute of Education, Bloomsbury, London, WC1H OAL, UK

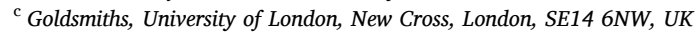

\section{A R T I C L E I N F O}

Number of reviews completed is 2

\section{Keywords:}

Attention Deficit Hyperactivity Disorder Developmental Coordination Disorder Attention

Motor

\begin{abstract}
A B S T R A C T
Background: The prevalence of motor impairment is high in ADHD, but we do not know if this stems from infancy.

Aims: 1) to compare the acquisition of motor milestones across three groups: Typically Developing (TD), Attention Deficit Hyperactivity Disorder (ADHD) and Developmental Coordination Disorder (DCD); 2) to determine the relationship between current motor ability and ADHD characteristics in children with ADHD.

Methods and procedures: The parents of children aged 8-16 years (ADHD, $\mathrm{N}=100$; DCD, $\mathrm{N}=66$; $\mathrm{TD}, \mathrm{N}=40$ ) completed three online questionnaires: Motor milestone questionnaire; Developmental Coordination Disorder Questionnaire (DCDQ’07) (concurrent motor ability); Conners 3 Parent Rating Scale Long Form (ADHD characteristics).

Outcome and results: When considered as a group, the ADHD group achieved motor milestones within a typical timeframe, despite concurrent motor impairments. Motor ability was not associated with ADHD characteristics. Latent Profile Analysis demonstrated that $56 \%$ of the ADHD group and $48 \%$ of the DCD group shared the same profile of motor milestone achievement, concurrent motor ability and ADHD characteristics.

Outcomes and conclusions: Unlike children with DCD, the motor impairment often observed in ADHD is not evident from infancy. It is also not part of the ADHD phenotype. Individual differences analysis demonstrated the broad heterogeneity of the ADHD phenotype.
\end{abstract}

What this paper adds?

This study investigated both concurrent and retrospective motor ability in children with ADHD. Children with DCD and typically

\footnotetext{
* Corresponding author at: School of Psychology, University of Surrey, Guildford, Surrey, GU2 7XH, UK.

E-mail addresses: jenna.lee@surrey.ac.uk (J. Lee), leighanne.mayall.14@ucl.ac.uk (L.A. Mayall), kathryn.bates.16@ucl.ac.uk (K.E. Bates), e. hill@gold.ac.uk (E.L. Hill), h.leonard@surrey.ac.uk (H.C. Leonard), e.farran@surrey.ac.uk (E.K. Farran).

1 Joint first authors.
} 
developing children were included as comparison groups. This is the first large-scale study to compare motor milestone achievement in children with ADHD, to children with DCD; we demonstrated that at a group level, unlike children with DCD, children with ADHD achieve early motor milestones within a typical timeframe. This suggests that motor impairments in ADHD emerge with development and are not necessarily present in infancy. This has important implications for intervention. We also took a novel, transdiagnostic approach to investigate heterogeneity in ADHD. Latent Profile Analysis identified four profiles of motor and ADHD characteristics. Three profiles consisted of predominantly one group (ADHD, DCD or TD). The fourth profile consisted of children with DCD and ADHD thus demonstrating the heterogeneity of these disorders, and their co-occurring characteristics.

\section{Introduction}

Attention Deficit Hyperactivity Disorder (ADHD) is a common neurodevelopmental disorder (3 \%-12 \%) (Polanczyk, de Lima, Horta, Biederman, \& Rohde, 2007), and is characterised by persistent problems with hyperactivity-impulsivity and/or inattention (DSM-5; American Psychological Association, 2013). Based on primary markers, ADHD is typically diagnosed in school aged children (Sonuga-Barke, Koerting, Smith, McCann, \& Thompson, 2011). Consequently, little is known about the interacting developmental processes that lead to $\mathrm{ADHD}$, the biggest clues to which are not necessarily the primary impairment, but subtle co-occurring impairments.

A co-occurring motor impairment is evident in children with ADHD, with approximately $50 \%$ meeting criteria for Developmental Coordination Disorder (DCD) (Athanasiadou et al., 2019; Brossard-Racine, Majnemer, \& Shevell, 2011; Farran et al., 2020; Kaiser, Schoemaker, Albaret, \& Geuze, 2015). DCD is an impairment in motor co-ordination which significantly affects daily living (DSM-5; American Psychological Association, 2013). Children with ADHD who meet the criteria for DCD have been reported to display fine and gross motor difficulties (Farran et al., 2020; Miyahara, Piek, \& Barrett, 2006; Pitcher, Piek, \& Hay, 2003) and have difficulties in handwriting (Brossard-Racine, Majnemer, Shevell, \& Snider, 2008). Further studies have investigated specific areas to demonstrate significant deficits for these children, relative to their typically developing (TD) counterparts, across motor speed, balance, postural stability and coordination (Amini, Hosseini, \& Akbarfahimi, 2018; Ayaz, Ayaz, \& Yazgan, 2010; Bucci, 2017; Sweeney et al., 2018).

Motor milestone achievement in children with ADHD has largely been neglected. Gurevitz, Geva, Varon, and Leitner (2014) measured motor milestone achievement using the Denver Developmental Screening Test (Frankenburg \& Dodds, 1967) at 0, 1, 3, 9 and 18 months in infants who later presented with ADHD traits $(\mathrm{N}=58)$, compared to a typically developing sample $(\mathrm{N}=58)$. Fine motor ability was not delayed in their ADHD sample. General motor delays were reported in $44.8 \%$ of the ADHD sample at 3 months, compared to $19 \%$ of the control sample. At 9 months gross motor delay was reported in $34.5 \%$ of the ADHD group compared to $13.8 \%$ of the control group, whilst the rates at 18 months were lower, albeit still significantly different, at $12.1 \%$ and $1.7 \%$ respectively. Importantly, the authors report a bipartile distribution, with motor milestone achievement falling at the extremes, and thus some children in this study showed particularly early gross motor milestone achievement. Two longitudinal cohort studies also support this finding; Jaspers et al. (2013) report an association between good gross motor skills in infancy and ADHD (Total N = 1816, including N = 419 ADHD), whilst (Lemcke, Parner, Bjerrum, Thomsen, \& Lauritsen, 2016) report that the majority of their ADHD sample (Total N $=62602$, including $\mathrm{N}=2034$ ADHD) showed typical motor development, but that there were associations with ADHD at both extremes of development. That is, walking independently before 11 months or after 15 months was associated with a later diagnosis of ADHD. Taken together, these results demonstrate wide heterogeneity in motor milestone achievement in ADHD. Furthermore, we do not know how this impacts later motor competency in childhood in ADHD.

There have also been several studies that have shown neurological differences in the brains of individuals with ADHD in comparison to TD controls. A meta-analysis of these studies demonstrated evidence for reductions in cerebral volume, cerebellum, prefrontal cortex and right caudate in individuals with ADHD (Valera, Faraone, Murray, \& Seidman, 2007). These volumetric reductions have also been implicated in the disturbed connectivity in the fronto-striatal-cerebellar neurocircuitry in ADHD (Castellanos \& Tannock, 2002; Durston et al., 2004; Hill et al., 2003; Valera et al., 2007). Overall, these changes in the brain are likely to contribute to the motor difficulties that some children with ADHD experience, given that the cerebellum is one of the main structures responsible for the execution of motor acts and the prefrontal cortex is responsible for motor planning (Crossman \& Neary, 2014).

The behavioural and neural studies above do not explain the prevalence of motor impairment in some, but not all, children with ADHD. Farran et al. (2020) used a neuroconstructivist framework to propose a developmental cascade hypothesis to explain variation across the disorder. Neuroconstructivism posits that the brain goes through a process of gradual specialisation and that this process is influenced by interactions between domains (e.g. motor, attention, language) across developmental time (see Karmiloff-Smith, 1998). Farran et al. (2020) demonstrated that 43 children aged 8 to 15-years with ADHD had achieved motor milestones in infancy at a typical time point regardless of whether current motor difficulties were exhibited. This suggested that the motor deficit observed in some children with ADHD emerges over developmental time. This differs from DCD where motor milestone achievement is delayed from infancy (Sumner, Leonard, \& Hill, 2016). Farran et al.'s (2020) developmental cascade hypothesis purports that the motor deficit in ADHD is a developmental consequence of tiny differences in development (e.g. in motor quality or attention) which, for some children have a negative cascading impact on motor development, whilst others are able to developmentally compensate for these differences, with little downstream impact on motor ability. If compensation is possible in this group, this has implications for intervention. Furthermore, because it is possible to measure the motor system and the attention system considerably earlier in childhood than other domains; these systems are particularly ripe for early intervention, which in turn has the power to strongly impact the developing system, while the brain has high plasticity (Heckman \& Masterov, 2007).

Farran et al.'s (2020) study is hampered by a low sample size. Furthermore, given the mixed findings regarding motor milestone achievement in ADHD reviewed above (Gurevitz et al., 2014; Jaspers et al., 2013), further research with a larger sample size and a 
measure of multiple motor milestones is required to determine whether and how motor milestone achievement is impacted in ADHD, and how this relates to the high prevalence of motor difficulties in this group. The current study was designed to replicate and extend Farran et al. (2020). The study used online questionnaires to collect data. Motor milestone achievement, current motor ability and ADHD characteristics in children with ADHD were measured. Retrospective motor milestone achievement is usually measured via questionnaire, and questionnaire measures of ADHD characteristics are also well validated and reliable. The measurement of current motor ability using an online questionnaire represents a cost-effective way of obtaining larger sample sizes than is typically possible with face-to-face testing sessions. Hence, this method was used to provide sufficient proof-of-concept data to test the hypothesis that motor milestone achievement in children with ADHD emerges within a broadly typical timeframe, and to determine how this relates to current motor ability and ADHD characteristics in this group. Furthermore, the current study built on previous findings by including a cross-syndrome comparison with DCD. The use of cross-syndrome comparison with DCD was designed to enable developmental differences between the motor impairment in ADHD and DCD to be determined. We hypothesised that, in line with Farran et al. (2020), motor milestone achievement is not delayed in children with ADHD but that, as found by Sumner et al. (2016), motor milestone achievement is delayed in children with DCD.

This study was also designed to determine the relationship between current motor ability and ADHD characteristics in the largest sample of children with ADHD to-date. Based on Farran et al. (2020), we hypothesised no relationship between motor ability and ADHD characteristics. Further, if a clear dissociation is established between the children in the DCD group and the children with ADHD who meet the criteria for "indication of DCD" on the Developmental Coordination Disorder Questionnaire (Wilson, Kaplan, Crawford, \& Roberts, 2007), for motor milestone achievement (i.e. delayed in DCD only), but not for current motor ability (i.e. impaired in DCD and ADHD), this will suggest that the motor impairment reported in some children with ADHD is not present from infancy. If this is the case, a motor impairment in ADHD has the potential to be attenuated or ameliorated through early intervention.

The range of variables collected also deemed this study suitable for Latent Profile Analysis (LPA). This technique ignores diagnostic group membership to examine the extent to which dependent variables cluster into distinct profiles of scores. We used LPA of our dependent variables to identify common profiles of scores and to model the probability of each individual belonging to a profile. This enabled us to determine whether the latent profiles identified recognised diagnostic group membership, and whether individuals with ADHD and individuals with DCD would be categorised as belonging to the same or different profiles. We hypothesised that the TD children would belong to a distinct profile relative to the DCD and ADHD groups, and that those children with ADHD and typical motor milestone achievement and/or low concurrent motor impairment (motor milestone and concurrent impairment based on parent report) would belong to a distinct profile from the DCD group.

\section{Methods}

\subsection{Participants}

Participants were split into three groups dependent on the child's diagnosis from a qualified clinical professional: ADHD (N $=100$ ), DCD $(\mathrm{N}=66)$, and TD $(\mathrm{N}=40)$. Exclusion criteria were pre-registered here (https://osf.io/8vs5k). For the ADHD group four participants were excluded because only the Conners 3 Parent Rating Scale (CPRS-3:L; Conners, 2008) had been completed, six participants were excluded for having a diagnosis of a learning disability or global developmental delay which negatively impacted IQ, and thus could have influenced motor ability (Smits-Engelsman \& Hill, 2012). A further four participants were excluded due to having Tourette's syndrome as this neurological condition is associated with motor problems. All responses reflected their child's behaviour when they had been off medication for a minimum of $24 \mathrm{~h}$. This left a final sample of 86 participants with a clinical diagnosis of ADHD, all of whom had a CPRS-3:L Global ADHD Index score over 65. The final sample included all subtypes of the disorder; predominantly hyperactive $(\mathrm{N}=14)$, predominantly inattentive $(\mathrm{N}=7)$ and combined $(\mathrm{N}=57)$. Eight parents reported 'Unknown' for subtype. Consistent with our study pre-registration, the ADHD group were also further split into an ADHD-high motor (N =7) and an ADHD-low motor ( $=79$ ) group. Parents completed the Developmental Coordination Disorder Questionnaire (DCDQ'07; Wilson et al., 2007). We used participant's scores on this to divide this clinically diagnosed group of children with ADHD into two motor categories. Specifically, a score of below 56 for children aged 8-years to 9-years 11-months, and a score of below 58 for children aged 10-years to 15-years (indicative of DCD), qualified these children for the ADHD-low motor group (see Materials for further details of the CPRS-3:L and DCDQ'07). All remaining children with ADHD were categorised as ADHD-high motor.

In the DCD group, one participant was excluded from the DCD group because only the CPRS-3:L had been completed. All remaining participants with DCD met the criteria for DCD on the DCDQ'07. Three participants were excluded due to the presence of learning difficulties (Smits-Engelsman \& Hill, 2012), and one participant was excluded due to having Tourette's syndrome. This left a final sample of $\mathrm{N}=61$ participants with a clinical diagnosis of DCD. Overall, 35 participants in the DCD group also met the criteria for ADHD on the CPRS-3:L (Global ADHD Index score >65).

In the TD group one participant was excluded because only the CPRS-3:L had been completed. Parents were asked to report whether their child had a diagnosis of a developmental disorder (with examples listed), and to state their child's diagnosis if they ticked 'Yes'. Based on our pre-registered exclusion criteria, that all TD children with a neurodevelopmental disorder would be excluded, one participant was excluded due to a neurodevelopmental diagnosis of Autism. Three participants were excluded for meeting criteria for DCD on the DCDQ'07, and a further five participants were excluded for meeting criteria for ADHD on the CPRS-3:L. This left a final sample of 31 individuals in the TD group. The mean age and gender of each group can be found in Table 1.

For the ADHD and DCD groups, parents/caregivers were asked to confirm diagnosis from a clinical/education practitioner. Confirmations included diagnostic letters from clinical leads, community paediatricians and paediatric neurologists, child development 
Table 1

Participant details.

\begin{tabular}{|c|c|c|c|}
\hline & Group & & \\
\hline & $\operatorname{ADHD}(\mathrm{N}=86)$ & $\operatorname{DCD}(\mathrm{N}=61)$ & $\mathrm{TD}(\mathrm{N}=31)$ \\
\hline Mean age in years (range) & $11.42(8.00-16.92)$ & $11.88(8.17-16.67)$ & $11.40(8.00-16.17)$ \\
\hline Gender F:M & $13: 73$ & $13: 48$ & $16: 15$ \\
\hline
\end{tabular}

service reports, neurodevelopmental specialist reports, child and adolescent psychiatry assessments, and cognitive assessment reports. Thirty parents in the ADHD group (35\%) and 21 parents in the DCD group (34\%) provided letters of confirmation of diagnosis. The remaining parents/caregivers confirmed via parental report of the clinical diagnosis. For children with a diagnosis of both ADHD and DCD, (ADHD: $N=2, \mathrm{DCD}: \mathrm{N}=5$ ) parents/caregivers were asked to confirm the primary diagnosis and this was used to determine group membership. Thus, although our group memberships are not derived from our own clinical assessments of the participants, we are confident from both parent report and confirmatory clinical diagnostic letters, that our groups had received clinical assessments to achieve their diagnoses.

Parents/caregivers of children with ADHD were recruited by sending an advert to the ADHD Foundation and other support/social media groups to be placed on their webpages. These groups were also asked to email the advert to their members. Parents/caregivers of children with DCD were recruited by sending an advert to the Dyspraxia Foundation and other support groups/social media groups to be placed on their webpages. These groups were also asked to email the advert to their members. The TD group was recruited via our existing networks of schools, and by social media. For all groups, adverts were placed on the Principle Investigator's website. Support groups, schools, charities, organisations and non-NHS affiliated clinics were also approached regarding recruitment for this project.

An ANOVA was performed on age with Group as the between participant factor (ADHD, DCD, TD). There was no significant difference in the mean age of the groups, $\mathrm{F}<1$. A Chi-squared test was performed on Group and Gender, $\left(\chi^{2}(2)=17.10, \mathrm{p}<.001, \Phi=\right.$ .31). Adjusted standardised residuals revealed the significant association was due to the high proportion of males in the ADHD group, which was not the case in the TD group.

\subsection{Materials}

These online questionnaires were completed using Qualtrics Software, Version 11.19 (Qualtrics, Provo, UT, 2019).

\subsubsection{Conners 3 parent rating scale (Conners, 2008)}

The Long Form of the Conners 3 Parent Rating Scale (CPRS-3:L) can be used for assessing ADHD symptoms and related behaviours in children aged 3-17 years. Comprised of 80 items, parents/caregivers rate their child's behaviours over the last month on a scale from Not True at All (Never)" to "Very Much True (Very Often)". It provides 14 subscales, including an ADHD Index with questions on Hyperactivity/Impulsivity and Inattention (within the CPRS-3:L, this factor is labelled 'Cognitive Problems'). The Conners Global ADHD Index subscale was used as the outcome measure for ADHD symptomology. This scale evaluates ADHD-related behaviour using a 10-item questionnaire. Conners Global ADHD Index raw scores are transformed into standardised $T$ scores based on age and gender with scores greater than 65 considered to be clinically significant. The scale has shown to be consistently effective at distinguishing between children with ADHD and children without ADHD (Conners, Sitarenios, Parker, \& Epstein, 1998). The questionnaire has good internal reliability (.75-.94) and the Conners Global ADHD Index test-retest reliability is high for both Hyperactivity/Impulsivity (.71) and Cognitive Problems/Inattention (.78) (Conners et al., 1998). We also attempted to collect data from teachers using the Long Form of the Conners 3 Teacher Rating Scale (CTRS-3). However, as this was an online study that required us to obtain teacher email addresses from parents, only 5 responses were collected, which was not a sufficient number to analyse.

\subsubsection{Parent questionnaire of the age of acquisition of key motor milestones (adapted from Sumner et al., 2016; based on Brouwer, van} Beijsterveldt, Bartels, Hudziak, \& Boomsma, 2006)

The motor milestone questionnaire asks parents/caregivers to retrospectively report the ages (in months) and/or dates of acquisition of ten motor milestones; lift head, turn back to belly, sit without support, crawl hands and knees, stand with assistance, stand without support, walk with assistance, walk without support, climb stairs, walk up/down stairs without support.

\subsubsection{Developmental coordination disorder questionnaire (Wilson et al, 2007)}

The DCDQ'07 asks parents/caregivers to rate their child's motor performance on a 5-point Likert scale relative to their peers. Comprised of 15 items the questionnaire is a screening tool for coordination disorders in children aged 5-15 years. The questionnaire assesses movement over three components; control during movement, fine motor/handwriting and general coordination. Children can fall into one of two categories: "indication of DCD" or "probably not DCD", dependent on age at the time of questionnaire completion and total scores across the three movement components (a total score of below 56 for children aged 8-years to 9-years 11-months, and a score of below 58 for children aged 10-years to 15-years, indicates DCD). Overall sensitivity for the measure is $84.6 \%$ and specificity is $70.8 \%$ when using age related cut-off scores and the MABC as a reference (Wilson et al., 2007). The questionnaire has a high overall internal reliability .95 (Rigoli, Piek, Kane, \& Oosterlaan, 2012) and .86 (Patel \& Gabbard, 2017). The internal reliability of the subscales is also high; Control During Movement (.94), Fine Motor/Handwriting (.88) and General Coordination (.85) (Rigoli et al., 2012). 
Table 2

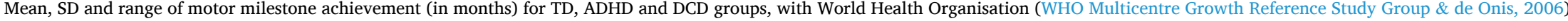
percentiles.

\begin{tabular}{|c|c|c|c|c|c|c|c|c|c|c|c|c|c|c|c|c|c|c|}
\hline & \multicolumn{2}{|c|}{$\begin{array}{l}\text { WHO Age in months at } \\
\text { which milestone } \\
\text { achieved }\end{array}$} & \multicolumn{4}{|c|}{ ADHD-Low motor Group } & \multicolumn{4}{|c|}{ ADHD-High motor Group } & \multicolumn{4}{|c|}{ DCD Group } & \multicolumn{4}{|c|}{ TD Group } \\
\hline & $\mathrm{M}(\mathrm{SD})$ & Range & $\mathrm{N}$ & $\mathrm{M}(\mathrm{SD})$ & $\%$ & Range & $\mathrm{N}$ & $\mathrm{M}(\mathrm{SD})$ & $\%$ & Range & $\mathrm{N}$ & M (SD) & $\%$ & Range & $\mathrm{N}$ & $\mathrm{M}(\mathrm{SD})$ & $\%$ & Range \\
\hline Lift head & & & 36 & $2.83(2.13)$ & & $0-12$ & 6 & $\begin{array}{l}2.58 \\
(2.339)\end{array}$ & & $0.5-7$ & 28 & $2.57(1.80)$ & & $0-8$ & 17 & $2.06(1.16)$ & & $0.5-4$ \\
\hline $\begin{array}{l}\text { Turn over from back to } \\
\text { belly }\end{array}$ & & & 38 & $4.90(1.29)$ & & $2-7$ & 3 & $3.00(1.00)$ & & $2-4$ & 24 & $5.70(2.33)$ & & $2-12$ & 22 & $4.02(1.37)$ & & $1-7$ \\
\hline Sit without support & $6.0(1.1)$ & $3.8-9.2$ & 38 & 7.09 (1.53) & 75th & $4-11$ & 5 & $5.90(1.88)$ & 50th & $3-8$ & 38 & $7.62(1.80)$ & 90th & $5-12$ & 23 & $5.91(1.11)$ & 50th & $2-7.5$ \\
\hline Crawl on hands and knees & $8.5(1.7)$ & $\begin{array}{l}5.2- \\
11.4\end{array}$ & 37 & $8.96(2.08)$ & 75th & $6-14$ & 4 & $7.75(1.50)$ & 25th & $6-9$ & 37 & $10.05(2.54)$ & 90th & $6-19$ & 25 & $8.44(1.66)$ & 50th & $4-11$ \\
\hline Stand with support & $7.6(1.4)$ & $\begin{array}{l}4.8- \\
11.4\end{array}$ & 49 & $10.08(2.81)$ & 95th & $6-21$ & 5 & $8.10(1.43)$ & 75th & $6.5-10$ & 42 & $10.99(2.95)$ & 97th & $5-22$ & 26 & $8.75(2.43)$ & 75th & $1-12$ \\
\hline Stand without support & $\begin{array}{l}11.9 \\
(1.9)\end{array}$ & $\begin{array}{l}6.9- \\
16.9\end{array}$ & 47 & $12.35(3.63)$ & 75th & $7-24$ & 6 & $9.67(2.58)$ & 25th & $6-12$ & 40 & $13.44(2.92)$ & 90th & $9-23$ & 26 & $\begin{array}{l}10.89 \\
(2.20)\end{array}$ & 50th & $7-16$ \\
\hline Walk with support & $9.2(1.5)$ & $\begin{array}{l}6.0- \\
13.7\end{array}$ & 50 & $11.89(3.12)$ & 95th & $8-21$ & 4 & $\begin{array}{l}10.75 \\
(1.89)\end{array}$ & 90th & $8-12$ & 44 & $13.36(3.23)$ & 99th & $9-24$ & 27 & $\begin{array}{l}10.89 \\
(2.20)\end{array}$ & 90th & $7-15$ \\
\hline Walk without support & $\begin{array}{l}12.1 \\
(1.8)\end{array}$ & $\begin{array}{l}8.2- \\
17.6\end{array}$ & 61 & $14.39(4.29)$ & 90th & $9-30$ & 7 & $\begin{array}{l}14.29 \\
(1.89)\end{array}$ & 90th & $13-17$ & 50 & $15.95(4.46)$ & 97th & $10-36$ & 29 & $\begin{array}{l}12.85 \\
(2.45)\end{array}$ & 50th & $9-18$ \\
\hline Climb stairs with support & & & 37 & $14.38(5.44)$ & & $2-30$ & 2 & $\begin{array}{l}12.00 \\
(1.41)\end{array}$ & & $11-13$ & 28 & $20.68(9.16)$ & & $8-42$ & 20 & $\begin{array}{l}11.80 \\
(2.12)\end{array}$ & & $8-15$ \\
\hline $\begin{array}{l}\text { Climb stairs without } \\
\text { support }\end{array}$ & & & 34 & $\begin{array}{l}23.16 \\
(10.75)\end{array}$ & & $3-60$ & 2 & $\begin{array}{l}17.00 \\
(2.83)\end{array}$ & & $15-19$ & 23 & $\begin{array}{l}35.15 \\
(18.69)\end{array}$ & & $12-72$ & 19 & $\begin{array}{l}18.21 \\
(3.34)\end{array}$ & & $14-24$ \\
\hline
\end{tabular}

Note: the ADHD-high motor group feature for descriptive purposes only; they did not feature as a group in the motor milestone analyses. 


\subsection{Design and procedure}

Ethical approval was obtained from the University Ethics Committee. All parents/caregivers viewed the information sheet online or as an email attachment prior to being directed to complete the consent form. All participants were volunteers and provided informed consent to participate in all elements of the study. Consent was gathered online.

Following consent, parents/caregivers of children with ADHD and/or DCD were asked to confirm that their child has a formal diagnosis and were given the option to upload an authorised confirmation letter/email to a secure server or to send this via email. In addition, for parents/caregivers of children with ADHD an option of providing the child's teachers information was presented. Teachers could then consent to take part in the study and complete the Conners' Teacher Rating Scale, also completed online.

Parents/caregivers also provided details of co-occurring disorders, school setting and year group. We asked parents to respond with reference to their child when they had refrained from taking ADHD-related medication for at least $24 \mathrm{~h}$ prior to completing the questionnaires. This could have been from memory if they preferred to keep their child medicated, but the memory must have been from a non-medicated period of more than $24 \mathrm{~h}$ within the last month. All parents responded while their child was off medication, as this has been shown to affect motor ability (Kaiser et al., 2015), and parents were asked to confirm this. Children with ADHD often have breaks from medication such as at the weekend, so this was not an unusual request for parents. All groups completed all questionnaires.

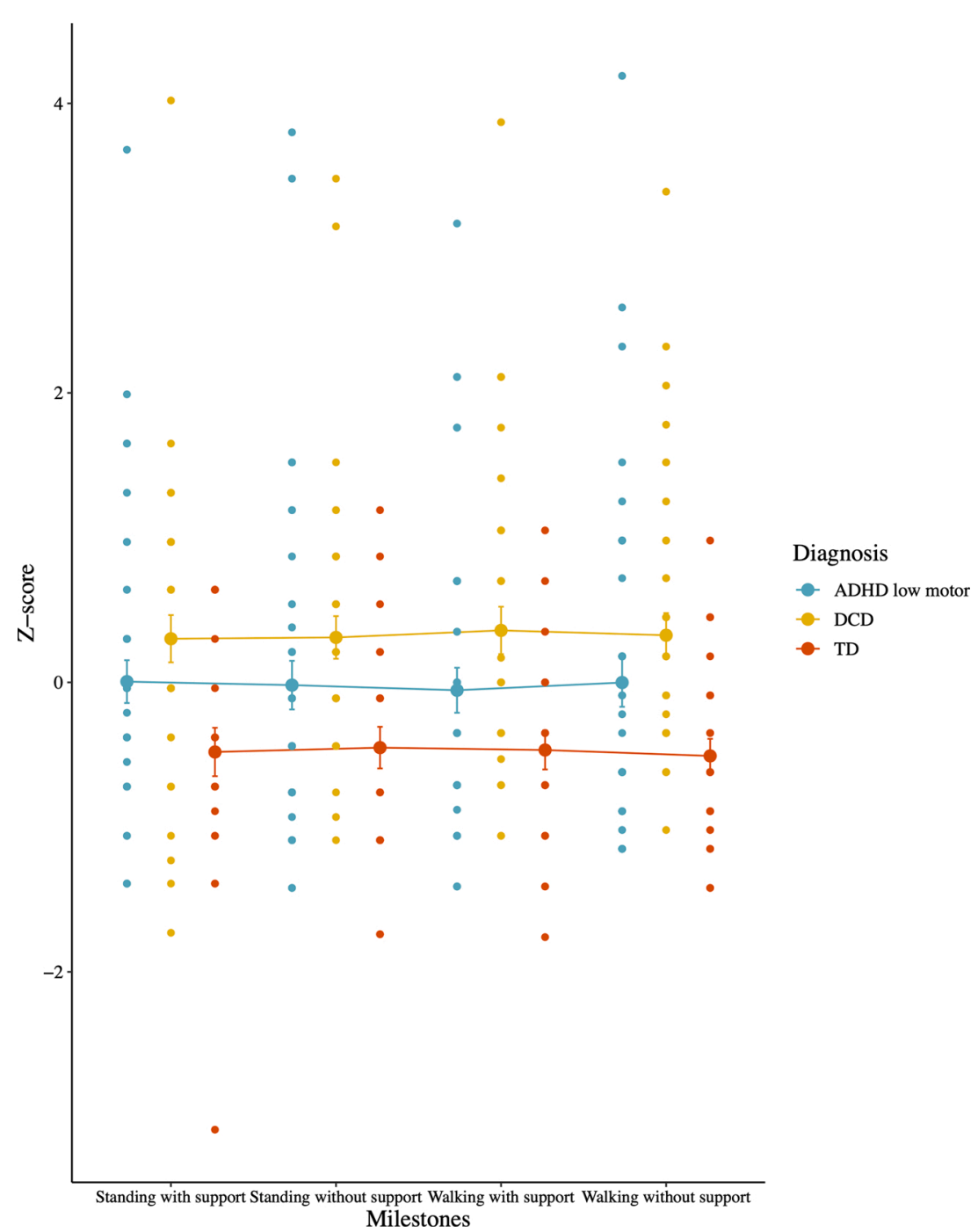

Fig. 1. Mean (standard error) Z-scores for four Motor Milestones, per group. 


\section{Results}

\subsection{Analysis plan}

Pre-registered (https://osf.io/8vs5k) analyses were: a Group by Milestone ANOVA, with month of achievement of the motor milestone as the dependent variable; and a correlational analysis between DCDQ'07 score and Conners Global ADHD Index score for the ADHD group. Analyses have been carried out as pre-registered, with the following exceptions. 1) For the ANOVA, we stated that we would divide the ADHD group into an ADHD-high motor and an ADHD-low motor. This division was based on the assumption that approximately $50 \%$ of participants with ADHD would fall into each ADHD group, consistent with the literature. The DCDQ'07 criteria for "indication of DCD" were used to divide the ADHD group into high and low motor groups. However, contrary to the literature, in our sample, there were only 7 who fell into the ADHD-high motor category. Thus, the ANOVA of motor milestone achievement was carried out first with these 7 children excluded, and then repeated with the ADHD-low motor and ADHD-high motor group treated as one group. 2) We stated that we would only run the ANOVA on motor milestone data that had reached $>75 \%$ response rate (missing data was anticipated due to the retrospective nature of this measure). However, this criterion was only reached for the 'walks without support' milestone. Instead, the four milestones with the most data (stand with assistance, stand without support, walk with assistance, walk without support) were used in the ANOVA. These four variables were available from $61 \%$ of the sample.

Additional exploratory analyses included: 1) a latent profile analysis of the dataset to explore group membership. 2) Correlations between DCDQ'07 score and Conners Global ADHD Index score were carried out for the DCD and TD groups. This was added for comparison with the equivalent pre-registered correlation analysis for the ADHD group. 3) We also included an ANOVA of group (ADHD, DCD, TD) on total DCDQ'07 scores, to investigate differences in concurrent motor ability across the groups. The data for this study can be accessed here:https://osf.io/7gqun/.

For the majority of variables, the data were not normally distributed (Kolmogorov-Smirnov, $\mathrm{p}<.05$ ). For correlational analyses, Spearman correlations were employed. For tests of group difference, because the sample sizes were large enough for the central limit theorem to apply (Field, 2013), parametric analyses were employed.

\subsection{Motor milestones}

The mean age of milestone achievement in months, for all 10 milestones, is presented in Table 2, along with the number of participants for each milestone, and the range of ages for each milestone. Five of the milestones examined can be compared to World Health Organisation (WHO) guidelines for when children typically reach a range of motor milestones (WHO Multicentre Growth Reference Study Group \& de Onis, 2006). Higher percentiles indicate later achievement of the milestone.

To remove effects of age across milestones, the age in months variable for motor milestone achievement was converted into zscores. ANOVA was carried out on the z-score of month of achievement, with the four milestones with the most data available as the within participant factor (standing with support, standing without support, walking with support and walking without support) and Group (TD, ADHD-low motor, DCD) as the between participant factor, as illustrated in Fig. 1. The sample sizes for this analysis were: ADHD-low motor: $\mathrm{N}=44$; DCD: $\mathrm{N}=39$; TD: $\mathrm{N}=25$. As expected, due to all z-scores having the same mean and standard deviation, there was no effect of the type of milestone, $F<1$. There was a main effect of group $\left(F(2,105)=6.34, p=.003, \eta_{p}^{2}=.11\right)$. Tukey pairwise comparisons demonstrated that this was due to the TD group achieving motor milestones at a younger age than the DCD group ( $p=$ .002). There were no differences between the ADHD-low motor group and the TD group $(p=.10)$ or DCD group ( $p=.19)$. There was no interaction between group and type of milestone $(F<1)$, suggesting that the effect of group was consistent across milestones. This

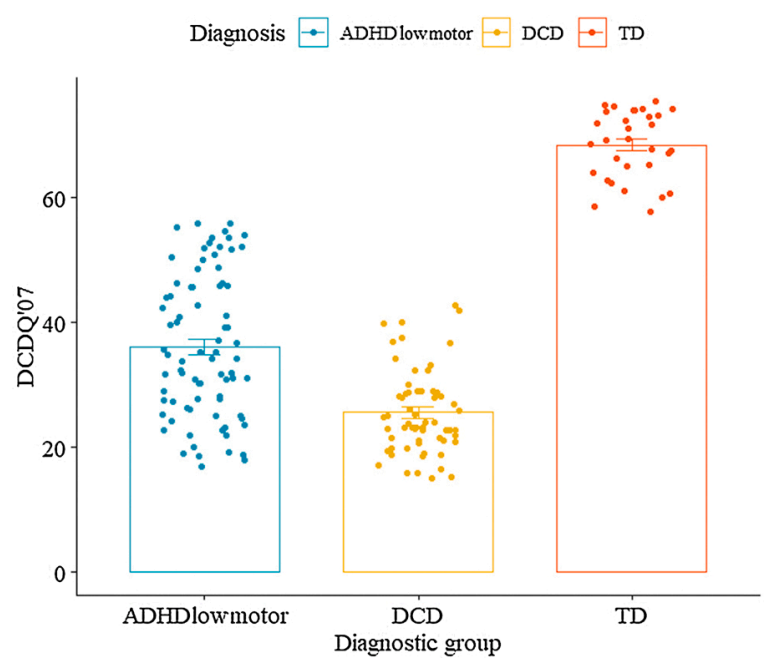

Fig. 2. Mean (standard error) total DCDQ'07 score, per group. 
pattern did not change when the analysis was re-run to include the whole $(\mathrm{N}=47)$ sample of the ADHD group.

\subsection{Motor ability}

ANOVA was carried out on total DCDQ'07 score, with Group (TD, ADHD-low motor, DCD) as the between participant factor. A significant difference was found across the groups, $F(2,168)=231.58, p<.001, \eta_{p}^{2}=.73$ (see Fig. 2). Post-hoc Tukey comparisons demonstrated that this was due to significant differences for all comparisons on account of stronger motor performance in the TD group, followed by the ADHD-low motor group and finally the poorest performance was observed in the DCD group ( $p<.001$ for all). This pattern did not change when the whole ADHD sample was included.

\subsection{Correlations between ADHD symptomology and motor ability}

To examine the relationship between ADHD symptomology (Conners Global ADHD Index score) and motor ability (DCDQ'07 score) in the sample, Spearman correlations were performed. To do this, in line with our pre-registration, the ADHD group were treated as one group (high motor and low motor, $\mathrm{N}=86$ ). A critical alpha of $p \leq .016$ was used to account for multiple comparisons. Correlations are displayed in Fig. 3. No correlations were significant ( $p>.016$ for all).

\subsection{Using Latent Profile Analysis to investigate individual differences in motor ability beyond diagnostic categories}

To examine the extent to which our dependent variables cluster into distinct profiles of scores, we employed Latent Profile Analysis across our whole sample (i.e. ignoring group membership). Variables included in the latent profile analysis were DCDQ'07 score, Conners 3 Global ADHD Index score and the motor milestone with the least missing data: walking without support (N = 147). The following criteria were applied to select the best model: 1) low Bayesian Information Criteria (BIC) and sample-adjusted BIC (SABIC) which indicates better model fit, i.e. less unexplained variance in the model, 2) significant Bootstrap Likelihood Ratio Test (BLRT) whereby a significant p value (alpha $<.05$ ) indicates a statistically significant difference between $k$ profiles and $k$ - 1 profiles, 3 ) entropy value above .90 where a value closer to 1 indicates higher probability of accurate class separation.
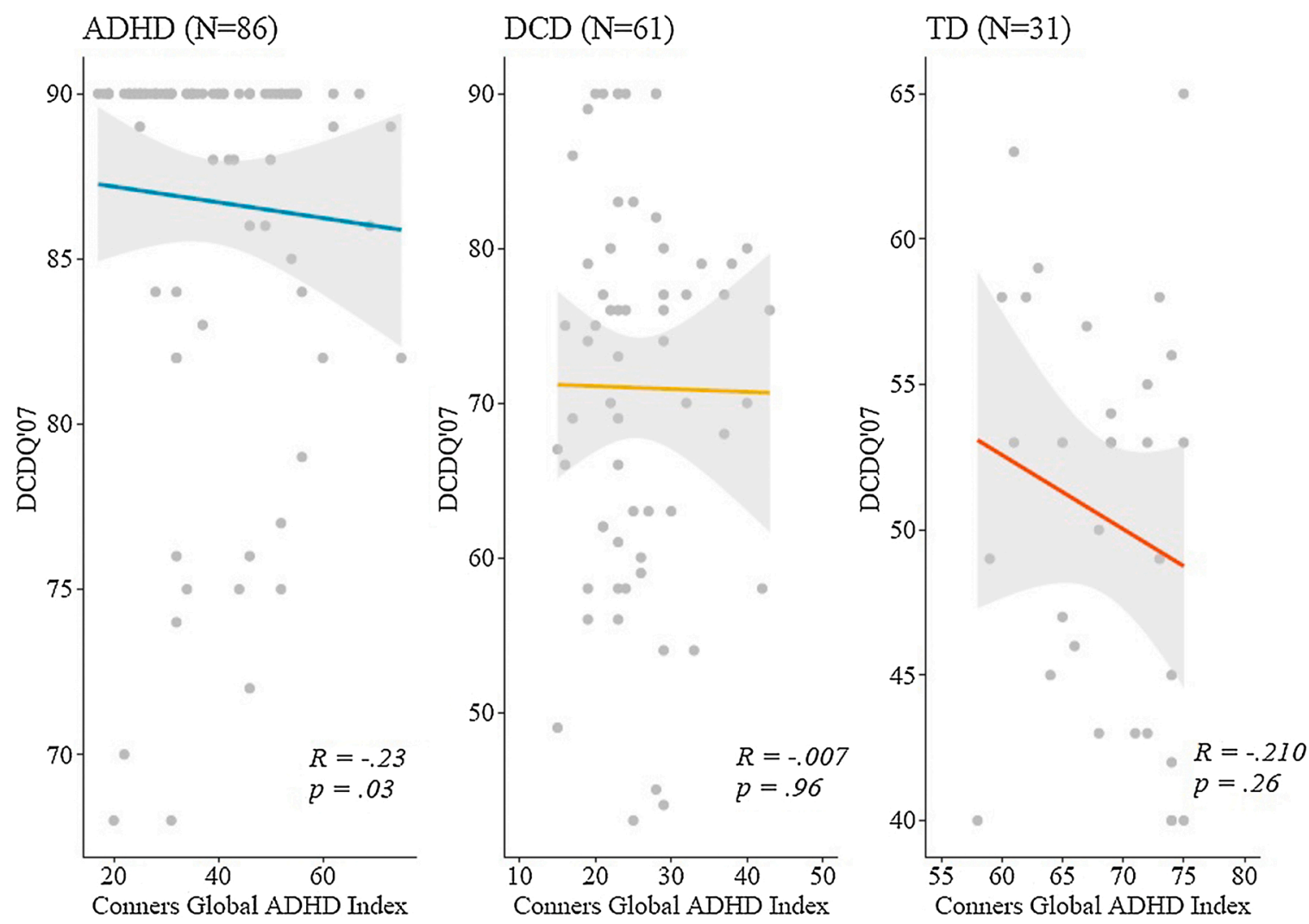

Fig. 3. Relationship between DCDQ'07 motor score and Connors Global ADHD Index score, per group. Scatter plots include linear best fit with confidence intervals. 


\subsubsection{Model fit}

To examine the optimum number of profiles, we assessed the fit of 1-6 profile models. Table 3 outlines the model fit statistics. The 5-profile model has the lowest BIC and second lowest SABIC values and the 6-profile model has the second lowest BIC and lowest SABIC respectively, thus indicating that these two models have the least unexplained variance. Entropy values are satisfactory for models including 1-6 profiles as the values are greater than .90, while the 5-profile model entropy value is higher than that of the 6-profile model. Each BLRT is significant $(\mathrm{p}<.05)$ suggesting better model fit as the number of profiles increased. While the 5-profile model appears the most accurate, consideration of the number of participants assigned to each profile shows that Profile 2 only includes 9 participants, i.e. $6 \%$ of the sample. As the sample size of this profile is so few, it was deemed that the 5-profile model is no more informative than the 4-profile model, therefore the 4-profile model was chosen as the final model.

\subsubsection{Profiles of ability}

Means and standard deviations of scores for each variable per profile can be found in Table 4. To consider ability across the profiles, typical motor milestone achievement was based on the WHO percentiles whereby the expected range of months of achieving walking without support is between 8.2-17.6 months. The mean of motor milestone achievement fell within the typical range for all profiles, however, a one-way ANOVA revealed a significant main effect of profile in motor milestone achievement $(F(3,143)=9.013, p<.001$, $\left.\eta_{p}^{2}=.16\right)$, whereby Tukey post-comparisons revealed significant differences in motor milestone achievement between profile 1 and $2(p$ $<.001)$, profile 2 and $3(p=.019)$, profile 2 and $4(p<.001$, all other $p s>.05)$. Distinct profiles of ADHD symptoms and motor ability were also supported by significant main effects of profile in the Conners Global ADHD Index $\left(F(3,143)=253.00, p<.001, \eta_{p}^{2}=.83\right)$ and DCDQ $\left(F(3,143)=327.77, p<.001, \eta_{p}^{2}=.87\right)$, respectively. Post-hoc Tukey comparisons revealed significant differences between profiles on each of the measures $(p s<.05)$ except for between Profile 1 and 3 on the Conners Global ADHD Index ( $p=.987)$.

Profile 1 included participants with a mean motor ability just below the DCDQ'07 cut-off for "indicative of DCD", high ADHD characteristics, and average motor milestone achievement (50th percentile). The second profile of participants is characterised by low motor ability, low ADHD characteristics and late motor milestone achievement (99th percentile). The third profile included those with low motor ability, high ADHD characteristics and relatively late motor milestone achievement (90th percentile). Finally, the fourth profile of participants included those with strong motor ability, low ADHD characteristics and average motor milestone achievement (50th percentile). The standardised means for each measure is plotted per profile in Fig. 4.

Next we examined the correlations between each variable (DCDQ'07, Conners Global ADHD Index, Motor milestone achievement) for each of the profiles to investigate whether the relationships among the variables differed across the profiles. The only significant correlation (critical alpha: $\mathrm{p} \leq$.017) revealed is a negative association between DCDQ'07 score and month of motor milestone achievement for Profile $3(r=-.319, p=.012)$, suggesting for participants in this profile, higher motor ability is associated with earlier motor milestone achievement. All other correlations between the three measures for each profile were not significant ( $\mathrm{p}>.017$ for all).

Finally, we were interested to see if the profiles reflected diagnostic groups. Interestingly, profiles 1, 2 and 4 contained 90-100 percent of participants from the same diagnostic group, but profile 3 contained a mixture of participants with a diagnosis of DCD and with a diagnosis of ADHD (see Fig. 5).

\section{Discussion}

The first aim of the current study was to investigate motor milestone achievement in children with ADHD and to compare this to a sample of TD children, and a cross-syndrome comparison group of children with DCD. The use of cross-syndrome comparison enabled us to identify whether any atypical motor development in the ADHD group was specific to the ADHD group, or common to other groups with motor impairment. We hypothesised that these two group would differ. That is, both groups would show motor impairment, yet motor milestone achievement would not be delayed in children with ADHD (in line with Farran et al., 2020), and would be delayed in children with DCD (in line with Sumner et al., 2016). This is broadly what we found. Unfortunately, we could not include an ADHD-high motor group in our analyses due to the small number of children who fell into this category. Nevertheless, we found that the DCD group reached motor milestones later than the TD sample, whilst the ADHD-low motor group reached motor milestones statistically at the same time as the TD group. Note, however, that the range of motor milestone achievement in the ADHD-low motor group was broad, to the extent that their motor milestone achievement also did not differ from that of the DCD group. Furthermore, the range of months of achievement of both the DCD and ADHD-low motor group overlapped with the range of ages considered to be in the typically developing range by the World Health Organisation (WHO Multicentre Growth Reference Study Group \& de Onis, 2006). Contrary to Gurevitz et al. (2014) and Lemcke et al. (2016), motor milestone achievement in the ADHD group was not bipartile.

Table 3

Model fit statistics for latent profile analysis models.

\begin{tabular}{|c|c|c|c|c|c|}
\hline Model & BIC & SABIC & Entropy & BLRT p value & $\mathrm{N}$ assigned to each Profile (P) \\
\hline 1-profile & 3381.751 & 3362.764 & 1 & - & $\mathrm{P} 1=147$ \\
\hline 2-profile & 3295.814 & 3264.169 & 0.925321 & $\mathrm{p}<.01$ & $\mathrm{P} 1=97, \mathrm{P} 2=50$ \\
\hline 3-profile & 3268.047 & 3223.744 & 0.929119 & $\mathrm{p}<.01$ & $\mathrm{P} 1=89, \mathrm{P} 1=29, \mathrm{P} 3=29$ \\
\hline 4-profile & 3221.82 & 3164.858 & 0.903241 & $\mathrm{p}<.01$ & $\mathrm{P} 1=27, \mathrm{P} 2=29, \mathrm{P} 3=62, \mathrm{P} 4=29$ \\
\hline 5-profile & 3199.667 & 3130.047 & 0.91072 & $\mathrm{p}<.01$ & $\mathrm{P} 1=27, \mathrm{P} 2=9, \mathrm{P} 3=57, \mathrm{P} 4=25, \mathrm{P} 5=29$ \\
\hline 6- profile & 3205.545 & 3123.267 & 0.902814 & $\mathrm{p}<.02$ & $\mathrm{P} 1=27, \mathrm{P} 2=9, \mathrm{P} 3=49, \mathrm{P} 4=7, \mathrm{P} 5=26, \mathrm{P} 6=29$ \\
\hline
\end{tabular}


Table 4

Means and standard deviation of scores on each measure per profile.

\begin{tabular}{|c|c|c|c|c|}
\hline Measures & $\begin{array}{l}\text { Profile } 1 \mathrm{~N}=27 \text { Mean } \\
(S D)\end{array}$ & $\begin{array}{l}\text { Profile } 2 \mathrm{~N}=29 \text { Mean } \\
(S D)\end{array}$ & $\begin{array}{l}\text { Profile } 3 \mathrm{~N}=62 \text { Mean } \\
(S D)\end{array}$ & $\begin{array}{l}\text { Profile } 4 \mathrm{~N}=29 \text { Mean } \\
(S D)\end{array}$ \\
\hline DCDQ & $55.00(8.46)$ & $24.00(5.61)$ & $28.33(7.14)$ & $68.55(5.25)$ \\
\hline Conners Global ADHD Index & $85.85(5.55)$ & $61.41(8.72)$ & $85.32(6.20)$ & $49.97(7.16)$ \\
\hline Motor milestone: Walking without support (month) & $12.96(3.97)$ & $17.38(5.18)$ & $14.86(3.47)$ & $12.84(2.45)$ \\
\hline $\begin{array}{l}\text { World Health Organisation }{ }^{1} \text { percentiles for Walking without } \\
\text { support (month) }\end{array}$ & 50 th & 99th & 90th & 50 th \\
\hline
\end{tabular}

1 (WHO Multicentre Growth Reference Study Group \& de Onis, 2006).

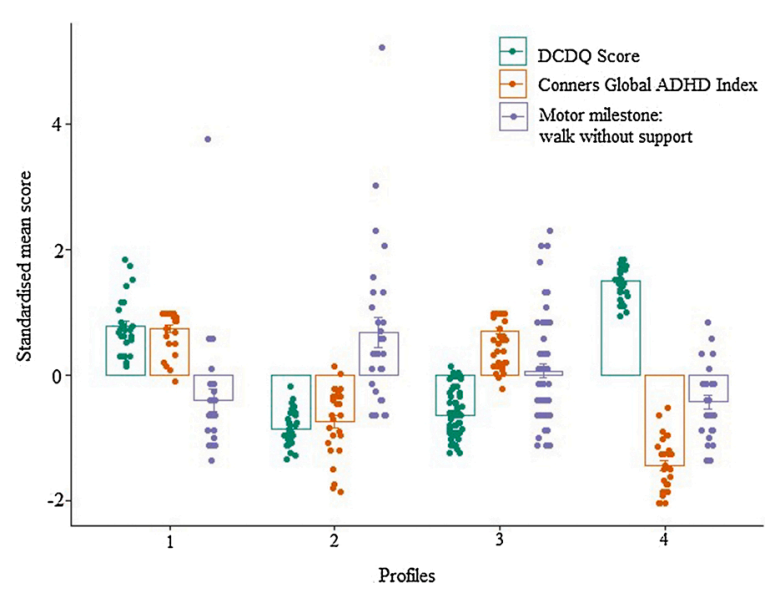

Fig. 4. Profile plot with $\mathrm{Z}$ score means (standard error) for each measure.

Generally, the broad range observed had a positive skew due to a longer tail for late motor milestone achievement than early motor milestone achievement. Thus, whilst motor milestone achievement in the ADHD-low motor group can be described as typical at the group level, there are broad individual differences. This was further illustrated by the Latent Profile Analysis (LPA), discussed below, in which the majority of the ADHD group were split across two of the four outcome profiles.

To provide a concurrent reference to the motor milestone data, we also investigated current motor ability in our groups using the DCDQ'07. Here we found differences across all three groups, with the strongest motor ability in the TD group, followed by the ADHDlow motor group, and finally the DCD group. The difference between the ADHD-low motor group and DCD group is somewhat surprising, because both groups met the criteria for "indication of DCD". This demonstrates, as shown in Fig. 2, that the motor deficit in DCD, as a group, is more severe than that observed in children with ADHD who have considerable motor difficulties. When considered at a group level, the motor milestone and concurrent motor ability data, suggest that the motor deficit often observed in ADHD differs from that observed in DCD with respect to its developmental emergence (i.e. motor milestones achievement is statistically delayed in DCD only), and level of severity in childhood. However, when considered at an individual level there are some parallels between DCD and ADHD. We return to this when discussing the LPA below, particularly profile 3 which included children with DCD and children with ADHD.

Our second aim stemmed from the high prevalence rate of motor difficulties in ADHD (Athanasiadou et al., 2019; Brossard-Racine et al., 2011; Farran et al. (2020); Goulardins, Marques, Casella, Nascimento, \& Oliveira, 2013; Kaiser et al., 2015; Tseng, Henderson, Chow \& Yao, 2004). We were interested in whether co-occuring motor difficulties in ADHD are part of the ADHD phenotype. (i.e. Is severity of ADHD characteristics associated with severity of motor deficit?). There was no correlation between ADHD characteristics and motor ability in any group. This replicates Farran et al. (2020) to suggest that the severe motor deficit observed in some children with ADHD is not part of the ADHD phenotype.

Further to our comparisons across diagnostic groups, we conducted an LPA to investigate individual differences in ADHD characteristics, motor ability and motor milestone achievement irrespective of diagnostic category. Forty percent of children with ADHD fell into profile 1 , which was characterised by motor ability which straddled the cut off for indication of DCD, and average motor milestone achievement. This profile is in line with the ADHD profile reported in Farran et al. (2020). Fifty-two percent of children with a diagnosis of DCD fell into profile 2, characterised by later motor milestone achievement as well as lower motor ability. This profile is also in line with previous evidence (Sumner et al., 2016). The TD group all fell into profile 4, which was characterised by typical performance for all three variables. Of note, whilst profiles 1, 2 and 4 largely consisted of one diagnostic group, profile 3 consisted of 61 $\%$ ADHD and $39 \%$ DCD. These children met the criteria for DCD and met the criteria for ADHD and demonstrated late (90th percentile) motor milestone achievement. Thus, contrary to the diagnostic group findings above, the LPA findings suggest that some children with ADHD who present with a motor impairment do reach motor milestones relatively late, and that these children do not differentiate on 


\section{Profile 1}

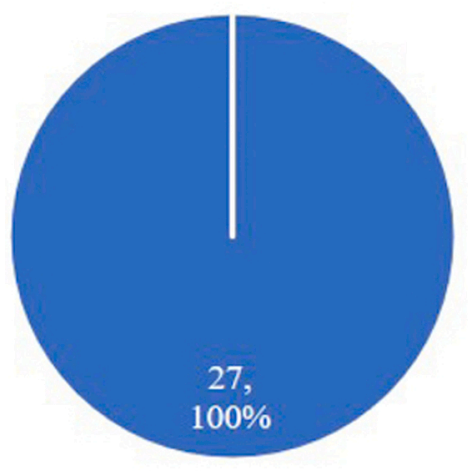

- ADHD

\section{Profile 3}

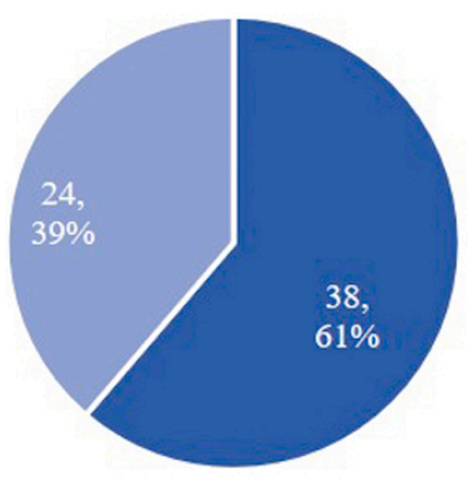

- $\mathrm{ADHD} \backsim \mathrm{DCD}$

\section{Profile 2}

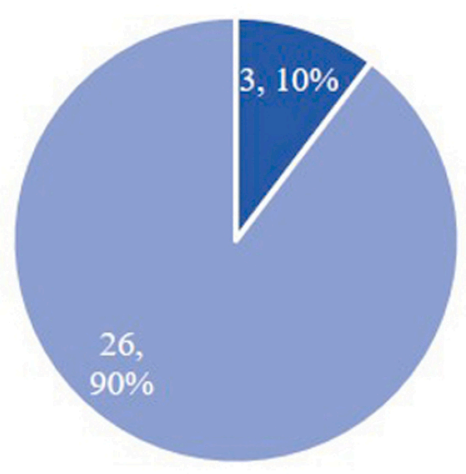

- $\mathrm{ADHD} \backsim \mathrm{DCD}$

\section{Profile 4}

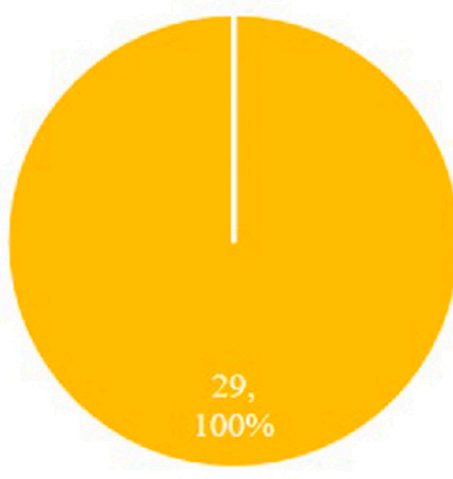

$\because \mathrm{TD}$

Fig. 5. Pie charts of percentage and number of participants with each diagnosis, per profile.

these variables from children with DCD. Note also, that this is the only profile in which motor milestone achievement and concurrent motor ability were associated. Equally, profile 3 also demonstrated high prevalence of ADHD characteristics in children with a primary diagnosis of DCD. The co-occurrence of DCD and ADHD is not uncommon and has been referred to as a distinct disorder: deficits in attention, motor control, and perception (DAMP; Gillberg, 2003). These children did not have a clinical diagnosis of DAMP, and it is important to note that a diagnosis of DAMP would not be solely based on parental report. The findings highlight the value of an individual differences approach to analysis, over and above comparisons between diagnostic groups, when examining motor abilities in neurodevelopmental disorders. That is, our data illustrate that a diagnosis of DCD or ADHD can reflect a spectrum of characteristics from a relatively pure disorder to one that co-occurs with characteristics of other neurodevelopmental disorders.

Considering the group analyses alongside the LPA analyses, while there were no group differences in motor milestone achievement between children with ADHD and either those with DCD or the TD children, the LPA demonstrated that $60 \%$ of children with ADHD presented with relatively late ( $\geq 90$ the percentile) motor milestone achievement alongside lower motor ability (Profile $2: 4 \%$ of the ADHD group; Profile 3: $56 \%$ of the ADHD group). Thus, whilst later motor milestone achievement is an early marker for DCD, this is not reliably the case for ADHD. Some children with ADHD were reported to have shown later motor milestone achievement (albeit within the typical range), but not all. That is, the children with ADHD who fell into LPA profile 1 had motor milestone achievement at the 50th percentile. Importantly, this does not necessarily protect against later motor impairment. For these children, some (in this 
study, the seven who did not meet the criteria for DCD) go on to develop motor abilities at a typical level, but many go on to develop severe motor difficulties. Thus, the developmental mechanism which protects some children with ADHD from developing a motor impairment cannot be detected via measures of motor milestone achievement.

Farran et al. (2020) suggested that the motor deficit in ADHD supports a developmental cascade hypothesis. In line with neuroconstructivism (Karmiloff-Smith, 1998), this states that the motor deficit in ADHD is a developmental consequence of subtle differences in development in early life in children with ADHD. In the current study, for some children these differences were already impacting motor development with reference to motor milestone achievement, but for others the subtle differences which gave rise to later motor difficulties were not detected. Further research is required to determine precisely what these subtle differences might be.

\subsection{Limitations and future research}

This online study was designed as a cost-effective way of obtaining relatively large sample sizes. As such, we were reliant on parent report throughout. Although we used measures that are known to be valid and reliable, we cannot rule out the potential of parental bias. Online data collection suited two of our primary variables very well (motor milestones and ADHD characteristics). For motor milestone achievement, because ADHD is not diagnosed until school age, retrospective parent report is the most suitable data collection method. Nonetheless, the missing data for this measure (due to parents forgetting or not having a record of this retrospective information), did reduce the sample size for analyses that used motor milestone data. For ADHD characteristics, the CPRS-3:L and CTRS-3, also parent/teacher report questionnaires, are seen as the Gold standard in the field. We were limited, however, with reference to teacher responses on the CTRS-3 because we were unable to obtain sufficient data and so could not include this measure in the analyses. Our 3rd primary variable, concurrent motor ability, we concede could have been a stronger variable had the data been gathered via a face to face testing session with the children. However, for the purposes of this proof-of-concept investigation, the DCDQ'07 served our purpose. As stated in the Methods section, the DCDQ'07 has high construct validity and correlates strongly with the MABC, a face to face motor assessment (Wilson et al., 2007).

A surprising finding, which was not consistent with the literature, was the low number of children in the ADHD group who did not meet the criteria for indication of DCD on the DCDQ'07. Given the high concurrent validity and sensitivity and specificity, of this measure, compared to the MABC as the reference measure (Wilson et al., 2007), it is unlikely that this reflects the measure employed. It is unlikely that this reflects a parental tendency to report high levels of impairment across different domains simply because their child has a neurodevelopmental disorder. If this was the case, we would have observed a correlation between ADHD characteristics and motor ability. One tentative explanation is that this might be an artefact of our advertising methods in which it was clear that we were interested in motor ability. This might have been more salient to parents of children with ADHD who had a motor difficulty than parents of children with ADHD who did not, thus biasing our (Miyahara et al., 2006)sample.

To truly delineate why some children with ADHD go on to develop severe motor deficits and others do not, investigations are required in which detailed attentional and motor variables are collected prospectively from infancy, with longitudinal follow-up into childhood. This would be possible via at-risk cohort studies. This will enable us to determine the subtle development differences in infancy which cascade developmentally to lead to motor impairment in childhood. It would also enable us to determine whether there are protective factors which enable some children to compensate for these developmental differences in infancy, thus protecting them from severe motor impairment in childhood.

\subsection{Conclusions}

Motor difficulties are very common in children with ADHD, but they are not necessarily evident from infancy. That is, children with ADHD broadly achieve motor milestones within a typical time frame. In contrast, children with DCD demonstrate both delayed motor milestone achievement and impaired concurrent motor ability. This contrast suggests different developmental pathways to impaired motor ability for these two groups. We also demonstrated that motor ability is not associated with ADHD characteristics, which suggests that motor impairment is not part of the ADHD phenotype. Our findings also highlight the importance of an individual differences approach to truly understand data; we were able to demonstrate that in addition to the group differences reported above, there were children with DCD and children with ADHD who displayed quite similar profiles.

\section{Funding}

This research was supported by The Waterloo Foundation (Grant number: 404-3611), University of Surrey Faculty Research Support Fund (Gran number: AC0607) and University of Surrey summer internship scheme.

\section{CRediT authorship contribution statement}

Jenna Lee: Investigation, Data curation, Writing - Introduction and method, Project administration. Leighanne A. Mayall: Data curation, Formal analysis, Writing - Introduction and Results. Kathryn E. Bates: Data curation, Formal analysis, Writing - Results, Visualization. Elisabeth L. Hill: Writing - review \& editing, Funding acquisition. Hayley C. Leonard: Writing - review \& editing. Emily K. Farran: Conceptualization, Methodology, Formal analysis, Data curation, Supervision, Funding acquisition, Writing Introduction, Results and Discussion, Writing - Review and Editing. 


\section{Declaration of Competing Interest}

None.

\section{Acknowledgements}

Thank you to Catriona McInnes, Rebeccah Webber and Alice Hamilton for help with recruitment and data curation. Thank you to the ADHD Foundation and the Dyspraxia Foundation for help with recruitment and to all of the families who took part.

\section{References}

American Psychiatric Association. (2013). Diagnostic and statistical manual of mental disorders (5th ed.). Arlington, VA: Author.

Amini, B., Hosseini, S., \& Akbarfahimi, N. (2018). Balance performance disorders and sway of the center of gravity in children with ADHD. Journal of Modern Rehabilitation, 12(1), 3-12. https://doi.org/10.32598/jmr.12.1.

Athanasiadou, A., Buitelaar, J. K., Brovedani, P., Chorna, O., Fulceri, F., Guzzetta, A., et al. (2019). Early motor signs of attention-deficit hyperactivity disorder: Systematic review. European Child \& Adolescent Psychiatry. https://doi.org/10.1007/s00787-019-01298-5 (0123456789).

Ayaz, A., Ayaz, M., \& Yazgan, Y. (2010). The relationship of motor coordination and ADHD. European Child \& Adolescent Psychiatry, 18, pS56. https://doi.org/ 10.1007/s00787-010-0117-5. -S56.

Brossard-Racine, M. B., Majnemer, A., Shevell, M., \& Snider, L. (2008). Handwriting performance in children with attention deficit hyperactivity disorder (ADHD). Journal of Child Neurology, 23(4), 399-406. https://doi.org/10.1177/0883073807309244.

Brossard-Racine, M., Majnemer, A., \& Shevell, M. I. (2011). Exploring the neural mechanisms that underlie motor difficulties in children with attention deficit hyperactivity disorder. Developmental Neurorehabilitation, 14, 101-111. https://doi.org/10.3109/17518423.2010.547545.

Brouwer, S. I., van Beijsterveldt, T. C., Bartels, M., Hudziak, J. J., \& Boomsma, D. I. (2006). Influences on achieving motor milestones: A twin-singleton study. Twin Research and Human Genetics, 9(3), 424-430. https://doi.org/10.1375/twin.9.3.424.

Bucci, M. (2017). Motor impairment in children with autistic spectrum disorder and in children with attention deficit hyperactivity disorder. Journal of Mental Health and Clinical Psychology, 1(1), 4-8. https://doi.org/10.29245/2578-2959/2018/1.1101.

Castellanos, F. X., \& Tannock, R. (2002). Neuroscience of attention-deficit/hyperactivity disorder: The search for endophenotypes. Nature Reviews Neuroscience, 3(8), 617-628. https://doi.org/10.1038/nrn896.

Conners, C. K. (2008). Conners third edition (Conners 3). Los Angeles, CA: Western Psychological Services.

Conners, C. K., Sitarenios, G., Parker, J. D., \& Epstein, J. N. (1998). The revised Conners' Parent Rating Scale (CPRS-R): Factor structure, reliability, and criterion validity. Journal of Abnormal Child Psychology, 26(4), 257-268. https://doi.org/10.1023/A:1022602400621.

Crossman, A. R., \& Neary, D. (2014). Neuroanatomy E-book: An illustrated colour text. Elsevier Health Sciences.

Durston, S., Pol, H. E. H., Schnack, H. G., Buitelaar, J. K., Steenhuis, M. P., Minderaa, R. B., et al. (2004). Magnetic resonance imaging of boys with attention-deficit/ hyperactivity disorder and their unaffected siblings. Journal of the American Academy of Child and Adolescent Psychiatry, 43(3), 332-340. https://doi.org/10.1097/ 00004583-200403000-00016.

Farran, E. K., Bowler, A., Hill, E. L., D’Souza, H., Mayall, L., Brady, D., et al. (2020). Is the motor impairment in Attention Deficit Hyperactivity Disorder (ADHD) a cooccurring deficit or a phenotypic characteristic? Advances in Neurodevelopmental Disorders. https://doi.org/10.1007/s41252-020-00159-6.

Field, A. (2013). Discovering statistics using IBM SPSS statistics. London, England: SAGE Publications.

Frankenburg, W. K., \& Dodds, J. B. (1967). The Denver developmental screening test. Journal of pediatrics, 71(2), 181-191. https://doi.org/10.1016/S0022-3476(67) $80070-2$.

Gillberg, C. (2003). Deficits in attention, motor control, and perception: a brief review. Archives of Disease in Childhood, 88(10), 904-910. https://doi.org/10.1136/ adc.88.10.904.

Goulardins, J. B., Marques, J. C. B., Casella, E. B., Nascimento, R. O., \& Oliveira, J. A. (2013). Motor profile of children with attention deficit hyperactivity disorder, combined type. Research in Developmental Disabilities, 34(1), 40-45. https://doi.org/10.1016/j.ridd.2012.07.014.

Gurevitz, M., Geva, R., Varon, M., \& Leitner, Y. (2014). Early markers in infants and toddlers for development of ADHD. Journal of Attention Disorders, 18(1), 14-22. https://doi.org/10.1177/1087054712447858.

Heckman, J. J., \& Masterov, D. V. (2007). The productivity argument for investing in young children. Review of Agricultural Economics, 29, 446-493. https://doi.org/ 10.1111/j.1467-9353.2007.00359.x.

Hill, D. E., Yeo, R. A., Campbell, R. A., Hart, B., Vigil, J., \& Brooks, W. (2003). Magnetic resonance imaging correlates of attention-deficit/hyperactivity disorder in children. Neuropsychology, 17(3), 496. https://doi.org/10.1037/0894-4105.17.3.496.

Jaspers, M., de Winter, A. F., Buitelaar, J. K., Verhulst, F. C., Reijneveld, S. A., \& Hartman, C. A. (2013). Early childhood assessments of community pediatric professionals predict autism spectrum and attention deficit hyperactivity problems. Journal of Abnormal Child Psychology, 41(1), 71-80. https://doi.org/10.1007/ s10802-012-9653-4.

Kaiser, M.-L., Schoemaker, M. M., Albaret, J.-M., \& Geuze, R. H. (2015). What is the evidence of impaired motor skills and motor control among children with attention deficit hyperactivity disorder (ADHD)? Systematic review of the literature. Research in Developmental Disabilities, 36, 338-357. https://doi.org/10.1016/ j.ridd.2014.09.023.

Karmiloff-Smith, A. (1998). Development itself is the key to understanding developmental disorders. Trends in Cognitive neuroscience, 2, 389-398.

Lemcke, S., Parner, E. T., Bjerrum, M., Thomsen, P. H., \& Lauritsen, M. B. (2016). Early development in children that are later diagnosed with disorders of attention and activity: a longitudinal study in the Danish National Birth Cohort. European Child \& Adolescent Psychiatry, 25(10), 1055-1066. https://doi.org/10.1007/ s00787-016-0825-6.

Miyahara, M., Piek, J., \& Barrett, N. (2006). Accuracy of drawing in a dual-task and resistance-to-distraction study: Motor or attention deficit? Human Movement Science, 25(1), 100-109. https://doi.org/10.1016/j.humov.2005.11.004.

Patel, P., \& Gabbard, C. (2017). Adaptation and preliminary testing of the developmental coordination disorder questionnaire (DCDQ) for children in India. Physical \& Occupational Therapy in Pediatrics, 37(2), 170-182. https://doi.org/10.3109/01942638.2016.1150383.

Pitcher, T. M., Piek, J. P., \& Hay, D. A. (2003). Fine and gross motor ability in males with ADHD. Developmental Medicine and Child Neurology, 45(8), 525-535. https:// doi.org/10.1017/S0012162203000975.

Polanczyk, G., de Lima, M., Horta, B., Biederman, J., \& Rohde, L. (2007). The worldwide prevalence of ADHD: A systematic review and metaregression analysis. The American Journal of Psychiatry, 164(6), 942-948. https://doi.org/10.1176/ajp.2007.164.6.942.

Rigoli, D., Piek, J. P., Kane, R., \& Oosterlaan, J. (2012). An examination of the relationship between motor coordination and executive functions in adolescents. Developmental Medicine and Child Neurology, 54(11), 1025-1031. https://doi.org/10.1111/j.1469-8749.2012.04403.x.

Smits-Engelsman, B., \& Hill, E. L. (2012). The relationship between motor coordination and intelligence across the IQ range. Pediatrics, 130(4), e950-e956. https:// doi.org/10.1542/peds.2011-3712.

Sonuga-Barke, E. J. S., Koerting, J., Smith, E., McCann, D. C., \& Thompson, M. (2011). Early detection and intervention for attention-deficit/hyperactivity disorder. Expert Review of Neurotherapeutics, 11, 557-563. https://doi.org/10.1586/ern.11.39.

Sumner, E., Leonard, H. C., \& Hill, E. L. (2016). Overlapping phenotypes in autism Spectrum disorder and developmental coordination disorder: A cross-syndrome comparison of motor and social skills. Journal of Autism and Developmental Disorders, 46(8), 2609-2620. https://doi.org/10.1007/s10803-016-2794-5. 
Sweeney, K. L., Ryan, M., Schneider, H., Ferenc, L., Denckla, M. B., \& Mahone, E. M. (2018). Developmental trajectory of motor deficits in preschool children with ADHD. Developmental Neuropsychology, 43(5), 419-429. https://doi.org/10.1080/87565641.2018.1466888.

Tseng, M. H., Henderson, A., Chow, S. M., \& Yao, G. (2004). Relationship between motor proficiency, attention, impulse, and activity in children with ADHD. Developmental Medicine and Child Neurology, 46, 381-388. https://doi.org/10.1017/S0012162204000623.

Valera, E. M., Faraone, S. V., Murray, K. E., \& Seidman, L. J. (2007). Meta-analysis of structural imaging findings in attention-deficit/hyperactivity disorder. Biological Psychiatry, 61(12), 1361-1369. https://doi.org/10.1016/j.biopsych.2006.06.011.

WHO Multicentre Growth Reference Study Group, \& de Onis, M. (2006). WHO motor development study: Windows of achievement for six gross motor development milestones. Acta Paediatrica, 95, 86-95. https://doi.org/10.1111/j.1651-2227.2006.tb02379.x.

Wilson, B. N., Kaplan, B. J., Crawford, S. G., \& Roberts, G. (2007). Developmental coordination questionnaire 2007 (DCDQ'07). Alberta Children Hospital. 